\title{
Clinical relapses and disease activity on magnetic resonance imaging associated with viral upper respiratory tract infections in multiple sclerosis
}

\author{
S Edwards, M Zvartau, H Clarke, W Irving, L D Blumhardt
}

\begin{abstract}
Background-Although the risk of clinical attacks of multiple sclerosis seems to be significantly increased with viral upper respiratory tract infections (URTI), serological evidence for the reported association remains controversial. In addition, although MRI is six to 10 times more sensitive than clinical exacerbations in indexing disease activity, any possible association between URTI and MRI activity has yet to be investigated.

Objectives-To examine the relation between URTI and disease activity, in multiple sclerosis patients participating in a placebo controlled trial of interferon $\beta-1 a$, as indexed both by clinical exacerbation rate and by the number and volume of gadolinium-diethylenetriaminepentaacetic acid (Gd-DTPA) enhancing lesions on MRI. "At risk" periods were defined around symptomatic URTI, with or without serological confirmation.
\end{abstract}

Results-The relative risk of clinical relapse for serologically unconfirmed symptomatic URTI was $2.1(p=0.004)$. Raised antiviral antibody titres conferred a relative risk of multiple sclerosis exacerbations that was 3.4 times higher than the "not at risk" periods (annual attack rates of $5.7 v 1.6$, respectively, $p=0.006$ ). There was no definite relation between the number or the volume of active lesions on MRI and either symptomatic or serologically defined at risk periods.

Conclusions-These results confirm the previously reported association between viral infections and multiple sclerosis exacerbations and indicate that the relative risk may be even higher when viral infection is serologically confirmed. However, the results, perhaps because of the confounding effects of interferon $\beta-1 a$, do not provide convincing evidence of increased blood-brain barrier breakdown or inflammation during periods of virally induced immune stimulation.

(F Neurol Neurosurg Psychiatry 1998;64:736-741)

Keywords: upper respiratory tract infections; viral serology; multiple sclerosis; magnetic resonance imaging

Studies of twins and populations emigrating from areas of low to high risk have provided evidence of the importance of environmental factors in the risk of acquiring multiple sclerosis. ${ }^{1-4}$ Whereas the initiating antigenic stimulus remains unknown, viral infection is considered by some authors to be the most likely factor in initiation of disease..$^{5-8}$ The finding that infections of the upper respiratory tract (URTI) are significantly associated with clinical exacerbations of multiple sclerosis, ${ }^{9-13}$ suggests that viruses may have a triggering role in the events that lead to blood-brain barrier breakdown or its inflammatory consequences. Both relapses and viral infections seem to be less common as the disease progresses. ${ }^{9}$ However, evidence of clinical URTI based on self reported symptoms may be less reliable than serial serological methods in providing evidence of viral exposure. Furthermore, as MRI is between six and 10 times more sensitive than clinical signs or symptoms in the assessment of disease activity, ${ }^{14-18}$ a stronger relation between URTI and active, ongoing acute changes on MRI might be expected. There have only been two previous attempts to link viral serology with multiple sclerosis relapses and these showed differing results, either a correlation with adenovirus in $\mathrm{CSF},{ }^{10}$ or an apparent increase in the titre of Epstein-Barr virus antibodies with attacks. ${ }^{11}$ No MRI data were included in these studies.

We report here the results of an investigation into the relation between symptomatic and serologically confirmed URTI on the one hand, and disease activity, as estimated both from the symptomatic attack rate and the number and volume of enhancing lesions on MRI, on the other.

\section{Methods}

PATIENTS

We collected data prospectively during the first 15 months of a randomised, placebo controlled trial of interferon $\beta$-1a (IFN $\beta-1 \mathrm{a}$ ) in 41 patients with either clinically definite (CDMS) or laboratory supported definite multiple sclerosis (LSDMS). ${ }^{19}$ There were 28 women and 13 men of median age 35 (range $21-50$ years), median EDSS score 3.5 (range 0-6.5), and median disease duration six years (range 1-28 years). In 21 patients the disease course could be defined as relapsing-remitting multiple sclerosis (RRMS) and in 20, secondary progressive multiple sclerosis (SPMS). RRMS was defined as CDMS or LSDMS with two or more acute exacerbations in the previous two years and a period of neurological stability without attacks for at least three months before study entry; SPMS was defined as CDMS with an initial relapsing and remitting course followed by a progressive deterioration of disability for at 
least six months and a deterioration of EDSS of at least one point during the past two years with or without superimposed acute attacks. $\mathrm{Pa}-$ tients with other relevant diseases were excluded. No patient had received any form of steroid treatment in the two months before entry and none had received previous immunosuppressive treatment. Patients were randomly assigned to either placebo, 6MIU, or $12 \mathrm{MIU}$ of interferon $\beta$-1a $(\operatorname{Rebif}(\mathrm{R}))$ three times weekly by subcutaneous injection.

The cohort was followed for a total of 2516 patient weeks, at monthly visits for the first nine months and then every three months for a further nine months. The patients were asked to record all new symptoms of multiple sclerosis exacerbations and any intercurrent infections in a specially designed diary. The neurological history and diary records were reviewed with the patient at the time of the neurological assessment at each visit.

SEROLOGICAL ASSAYS

Panels of 10 serum samples from each patient taken at monthly intervals until month 9 were assayed for the presence of antibodies to influenza A, influenza B, respiratory syncytial, adenoviruses, cytomegaloviruses, and enteroviruses, by standard complement fixation tests. Serum samples were stored at $-80^{\circ} \mathrm{C}$ until use.

Each patient panel was thawed and tested against all six viral antigen preparations in the same assay. Initial screening was performed at a serum dilution of 1 in 32 . If all 10 serum samples within the panel were negative for antibodies to a particular antigen at the screening dilution, no further testing was performed. If the pattern of reactivity within the panel changed from negative to positive, then the four samples temporally spanning this change were retested and titred accurately by twofold dilutions. Declining titres of antibody - that is, when the pattern of reactivity at the screening dilution changed from positive to negativewere not investigated further. If all samples within a panel were positive at the screening dilution, samples taken at 0 , three, six, and nine months were titred to determine if a fourfold or greater rise had taken place during the year.

Infection with a particular virus was defined serologically as a fourfold or greater rise in specific antibody titre in two samples (referred to as samples A and B, see later) taken one month apart.

MRI PROTOCOL

A 1.5T MRI system (Magnetom SP; Siemens Medical Systems) was used to obtain GdDTPA enhanced, T1 weighted 3D acquired MP-RAGE (magnetisation prepared rapid acquisition gradient echo) images. We utilised gradient recalled echo sequence with a small flip angle, performed with a three dimentional Fourier transform acquisition technique after magnetisation prepared, $180^{\circ}$ inversion pulses. The sequence parameters were $\mathrm{TR}=10, \mathrm{TE}=4$, $\mathrm{TD}=100, \mathrm{TI}=300$, flip angle $=10^{\circ}$, matrix $=192 \times 256$, field of view $=250 \mathrm{~mm}$, slab $=180 \mathrm{~mm}$, including 128 sagittal partitions with an effective slice thickness of $1.406 \mathrm{~mm}$.
The acquisition time was 5.25 minutes. Reproducible head positioning was performed by external landmarks (outer canthus of eye to tragus; acanthion to tragus; interpupillary line) and internal landmarks (line parallel to inferior border of genu of corpus callosum to inferior border of splenium of corpus callosum).

DATA ANALYSIS

The "at risk" and "not at risk" periods for multiple sclerosis exacerbations/MRI disease activity were defined differently depending on which viral correlate (clinical URTI or serological antibody titre rise) was being analysed.

The at risk period associated with symptomatic URTI was defined as a period from 14 days before until 14 days after the first symptom of each reported URTI. All other periods were considered not at risk as defined by viral URTI symptomatology.

As antibody production may not occur for up to two weeks after the time of infection, ${ }^{20}$ the at risk period for serologically defined infections was defined as the interval from two weeks before sample A until two weeks after sample B-that is, an at risk period of eight weeks. All periods before this rise in antibody titre were considered not at risk as defined by antibody titre rise. Periods after the at risk period were not considered, as once the titres had risen it would be difficult to identify a further viral exposure.

An exacerbation of multiple sclerosis was defined as the development of a new neurological symptom or symptoms, or worsening of an existing symptom or symptoms, attributable to multiple sclerosis, which lasted more than 24 hours. ${ }^{19}$ Attacks were separated into two groups defined as either mild, or moderate/severe according to the change in the Scripps neurological rating scale (SNRS) (0-7 for mild, 8-14 for moderate, $>14$ for severe) or effects on activity of daily living (ADL) (no effect for mild, significant for moderate, admission to hospital due to multiple sclerosis for severe). The symptoms recorded in the diaries which were accepted as evidence of infection were flu or cold, or, combinations of two or more of malaise, sore throat, muscle aches and pains, running or blocked nose, or fever. Only four patients with infections reported a fever and on none of these occasions was there a temporal association with a multiple sclerosis exacerbation. Symptoms of gastrointestinal or urinary tract infections were not included in this analysis.

Available data on vaccinations allowed two groups to be defined: those who had influenza $B$ vaccinations during the period from October 1994 until January 1995 (eight patients) and those who did not (26 patients).

MRI ANALYSIS

T1 weighted postcontrast images were reviewed by two observers who were blinded to the clinical details. The analysis was carried out with a manual thresholding technique using Analyze image analysis software on a Silicon Graphics workstation. Hyperintense white matter lesions were identified independently by 
Table 1 Clinical exacerbations of multiple sclerosis associated with URTI and with serological rise in antibody titres

\begin{tabular}{lcll}
\hline Period & Patient-weeks (n) & Attacks (n) & Attack rately \\
\hline All symptomatic URTI: & & & \\
$\quad$ At risk & 430 & 27 & 3.3 \\
$\quad$ Not at risk & 2086 & 65 & 1.6 \\
Serologically proved viral infection: ${ }^{\star \star}$ & 64 & 7 & 5.7 \\
$\quad$ At risk & 1552 & 50 & 1.7 \\
$\quad$ Not at risk & &
\end{tabular}

${ }^{\star} \mathrm{p}=0.004$, odds ratio $2.0(95 \%$ CI $1.3-3.2)$.

$\star \star \mathrm{p}=0.006$, odds ratio $3.4(95 \%$ CI $1.5-7.8)$

URTI=Upper respiratory tract infection.

There are less patient-weeks in the serological data as blood was only collected monthly for the first 9 months.

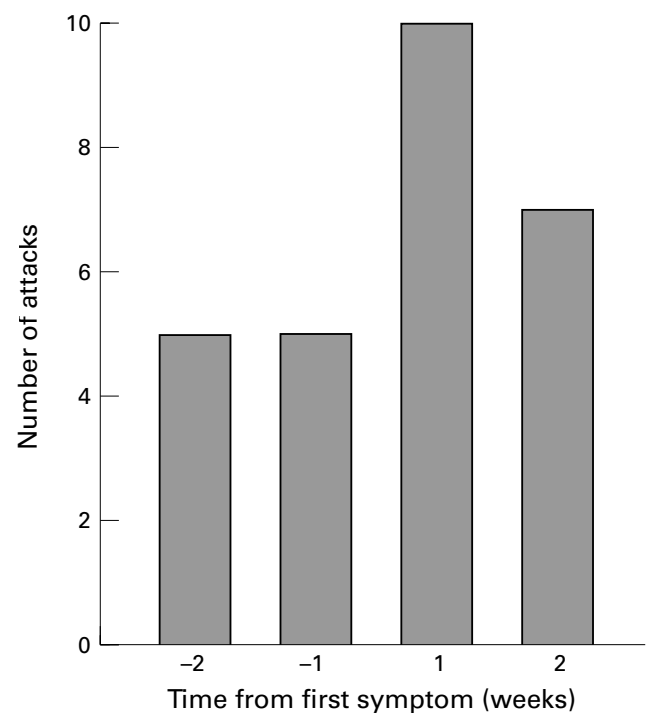

the two observers, or, where discrepancies occurred, were reconciled after review of the data. A threshold value for the voxel T1 signal intensity in normal appearing white matter in one of the slices which also contained the enhancing tissue to be quantified, was defined as the mean plus 3 SD for all the voxels within the region sampled. A second three dimensional region of interest (ROI) which contained the Gd-DTPA enhancing lesion to be measured and some surrounding normal appearing white matter was then defined and all voxels with an intensity exceeding the threshold for normal appearing white matter were then counted to obtain the volume of enhancing tissue for that lesion. This method was then repeated for all lesions in each brain.

Repeated threshold determinations were made from areas of normal appearing white matter and applied to ROIs containing enhancing areas. The intraobserver error (variance) for these two manoeuvres, expressed as variance (the average of the sum of the squared deviations from the mean of the sample divided by the degrees of freedom), was $7.5 \%$.

The numbers of enhancing lesions and the estimated volumes of the enhancing tissue were measured in all at risk scans and in a randomly selected sample of not at risk scans, as defined both by clinical URTI and antibody titre rise.

STATISTICAL ANALYSIS

The differences in estimated disease activity (from both clinical and MRI data) at times of at risk periods (as defined clinically or serologically) compared with not at risk periods, were examined by $\chi^{2}$ test (two tailed), the results being expressed as an odds ratio (Haldane's correction) with $95 \%$ confidence intervals (95\% CIs) (Arcus software version 2.15a). Differences between the vaccinated and nonvaccinated group were analysed in a similar fashion.

\section{Results}

SYMPTOMATIC VIRAL INFECTIONS AND EXACERBATIONS OF MULTIPLE SCLEROSIS

A total of 114 URTIs were reported by 34 of 41 patients $(83 \%)$ during the 15 month period of observation (2516 patient-weeks), resulting in an average annual infection rate of 2.4 . Nineteen of 114 URTIs (17\%) were associated with exacerbations of multiple sclerosis. Twenty seven of 92 multiple sclerosis (29\%) exacerbations occurred during at risk periods as defined by symptomatic URTI.
Histogram of number of multiple sclerosis attacks within each week of the clinically defined at risk period.

At risk periods around symptomatic URTIs (relative attack rate 3.3) were associated with a twofold increase in exacerbations $(\mathrm{p}=0.004$, OR 2.0, CI 1.3-3.2) compared with not at risk periods (relative attack rate 1.6, table 1). Within the at risk period, most of the attacks occurred during the first two weeks after the onset of clinical URTI (fig). Patients with RRMS and SPMS had similar relative risks. The patients in the study had either moderate or severe attacks. The severity of the attacks of multiple sclerosis was moderate in six of 27 attacks $(22 \%)$ that occurred during the at risk periods and in 12 of $65(18 \%)$ attacks during the not at risk periods $(\mathrm{p}=0.9$, OR $0.79, \mathrm{CI}$ $0.26-2.39)$.

VIRAL SEROLOGY AND CLINICAL MULTIPLE SCLEROSIS ATTACKS

Sixty four clinical URTIs were reported during the 10 month period in which monthly blood samples were obtained from the patients. Eight of $64(12.5 \%)$ clinical URTIs were accompanied by a rise in antibody titres asymptomatic, table 2). The at risk periods defined by positive serology were associated with a 3.4-fold increase in clinical multiple sclerosis exacerbations (equivalent to an annual attack rate of 5.7), compared with periods not at risk $(\mathrm{p}=0.006$, OR 3.4, CI 1.5-7.8, table 1 ). The exacerbation rate of multiple sclerosis during periods not at risk, as defined by serology, was similar to the rate during the not at risk periods defined by symptomatic URTIs (table 1).

Patients who reported vaccination with influenza vaccine in late 1994 (eight out of 41) did not significantly differ from those who did not have vaccination for rates of symptomatic URTIs ( $2.1 v 2.6)$, multiple sclerosis exacerbations (3.0 $v 2.8)$, or significant increases in antibody titres $(0.13 v 0.27)$ during 1995. to one of two viruses (one rise of titre was 
Table 2 URTI, clinical attacks, and viral serology

\begin{tabular}{llll}
\hline & $\begin{array}{l}\text { Raised } \\
\text { titres }\end{array}$ & $\begin{array}{l}\text { Symptmatic } \\
\text { URTI }\end{array}$ & $\begin{array}{l}\text { Clinical multiple } \\
\text { sclerosis attacks }\end{array}$ \\
\hline Influenza B & $6^{\star}$ & 7 & 4 \\
Adenovirus & $1 \dagger$ & - & 1 \\
RSV & $1 \ddagger$ & 1 & 2 \\
Total & 8 & 8 & 7
\end{tabular}

* One patient had two clinical URTIs during the single rise of antiviral antibody titre.

†One patient had a rise of antibody titres with no symptoms of URTI.

¥One patient had two multiple sclerosis attacks during the single rise of antiviral antibody titre.

RSV=respiratory syncytial virus; URTI=upper respiratory tract infection.

SEROLOGICALLY ESTABLISHED INFECTION AND

MULTIPLE SCLEROSIS DISEASE ACTIVITY ON

GD-DPTA ENHANCED MRI

Serological confirmation of a viral infection was obtained in eight of the 41 patients (19.5\%). Brain MRI acquired during the at risk periods defined by the eight titre rises, contained a mean of 1.125 lesions (range $0-6$ ) compared with 0.69 lesions/scan (range 0-4) during not at risk periods $(\mathrm{p}=0.44)$.

Five of the eight patients showed no significant enhancing load in either at risk or not at risk periods (despite clinical exacerbations in two). The other three patients showed greater volumes of enhancing tissue on scans during serological at risk periods, all of which were associated with clinical exacerbations. Their three scans contained a total of 1206 enhancing voxels. This was compared with the 350 enhancing voxels on 16 scans randomly sampled from not at risk periods in the same patients $(p<0.0001, O R=4.5,95 \%$ CI 4.0 5.1).

SYMPTOMATIC URTI AND MULTIPLE SCLEROSIS DISEASE ACTIVITY ON GD-DTPA ENHANCED MRI Symptomatic URTIs coincided with neuroimaging in 14 of the 41 (34\%) patients. We compared MRI during times of symptomatic URTIs (at risk) with MRI during asymptomatic periods (not at risk) in these patients. There was no increase in disease activity during defined periods of symptomatic URTI, whether measured by the volume or the number of Gd-DTPA enhancing multiple sclerosis lesions.

\section{Discussion}

The initial event in the pathology of a new multiple sclerosis plaque is considered to be blood-brain barrier breakdown leading to perivenular oedema, $\mathrm{B}$ and $\mathrm{T}$ cell infiltration, and increased plasma cells and macrophages. $^{2122}$ The inflammatory process continues with the increased presence of phagocytic cells, such as foamy macrophages, around the areas of myelin breakdown, associated with increased astrocyte and oligodendrocyte precursors and depletion of the myelin producing mature oligodendrocytes. ${ }^{7}$ The putative roles for viruses include a direct cytopathic effect as seen in progressive multifocal leukoencephalopathy, ${ }^{23}$ and viral stimulation or modification of the host immune system. Virally induced immune mediated demyelina- tion may occur through release of sequestered antigens such as myelin basic protein, increased expression of HLA molecules by cytokine induction, incorporation of a host component into a virus, disruption of the immune system leading to imbalance and autoimmunity, or molecular mimicry between viral and host protein sequences leading to autoimmunity. Alternatively viruses may act as the presumed primary event, causing increased permeability of the blood-brain barrier by increasing cytokine induced adhesins or by recognition of an endothelial epitope by virally induced peripheral blood T cells. ${ }^{8}$

Many viruses have been implicated in the pathogenesis of multiple sclerosis, including measles, ${ }^{24}$ parainfluenza, ${ }^{25}$ canine distemper, ${ }^{26}$ SV $5,{ }^{27}$ retroviruses such as HTLV- $1,{ }^{28}$ Epstein-Barr, ${ }^{29}$ coronaviruses, ${ }^{30}$ adenoviruses, ${ }^{10}$ herpes simplex, and reoviruses. ${ }^{31}$ The fact that no single virus has been consistently linked to multiple sclerosis implies that there may not be a specific multiple sclerosis agent and that several viruses may be capable of triggering abnormal immune activity, or that the cooperation of two viruses may be required to produce the pathogenic demyelination. The second hypothesis is supported by the finding that herpes simplex virus enhanced retroviral expression in a leptomeningeal line from a multiple sclerosis patient. ${ }^{32}$

In the present study, the definition of at risk periods associated with URTI was different from that used by Sibley et al (from two weeks before to five weeks after an URTI) or by Panitch (from one week before to five weeks after an URTI). ${ }^{9}{ }^{12}$ We accepted the definition of the beginning of the period as two weeks before the onset of clinical URTI, ${ }^{9}$ as it is not always easy to define the exact onset day, and because virus shedding can sometimes occur as early as 13 days before the onset of clinical symptoms. ${ }^{33}$ However, we limited the end of the at risk period to two weeks after the onset of clinical URTI, as viral replication is most unlikely to persist beyond this period. Andersen et al were unable to reproduce the results of Sibley et al by using the same definition of at risk period (two weeks before to five weeks after the onset of symptomatic URTI). ${ }^{910}$ When the at risk period was reduced to four weeks (one week before and three weeks after the onset of symptomatic URTI) a weak relative risk of exacerbation of 1.32 was obtained $(p=0.0477)$.

Despite these differences, our data support earlier reports that URTI are a significant risk factor for multiple sclerosis exacerbations. We found that $29 \%$ of multiple sclerosis exacerbations occurred during at risk periods as defined by symptomatic attacks. This is very similar to the rates of $27 \%^{9}$ and $32 \%$ previously reported. ${ }^{11}$ Further, the significantly raised relative risk $(R R=2.1)$ of multiple sclerosis exacerbations during at risk periods associated with symptomatic URTI in the present study compares closely with earlier reports (RRs of 2.8 and 2.5). ${ }^{9}{ }^{12}$

On the other hand, most URTIs do not precipitate exacerbations of multiple sclerosis. The $17 \%$ of URTIs that we found were associated 
with attacks compares with rates of $15 \%$ and $6.8 \%(3.1 \%$ for highly disabled patients and $10.5 \%$ for those with little disability) in previous reports. ${ }^{9} 11$

Our serological data, although limited by the availability and complexity of tests for common upper respiratory tract viruses, supports the hypothesis that several different viruses may be implicated in triggering multiple sclerosis attacks. We found a higher relative risk of exacerbations ( $R R=3.4)$ when URTIs were serologically confirmed, although we acknowledge that most attacks were serologically negative. It is uncertain whether a wider search for viruses would have resulted in an even stronger serological association, or whether other agents or triggering factors are responsible for initiating most attacks.

Our results do not support an obvious effect of influenza vaccination on the rate of either attacks of multiple sclerosis or URTI, despite the fact that four of seven of the relapses were associated with positive serology to influenza B. This finding is consistent with the results of a recent prospective study of influenza vaccination in multiple sclerosis. ${ }^{34}$ Despite these results this area deserves further investigation as vaccination could be an important preventive option if the range of viral pathogens associated with multiple sclerosis exacerbations is restricted.

Our patients were participating in a double blind placebo controlled trial of IFN $\beta-1$ a and, as yet, we do not know which patients were on active treatment. However, we have assumed from the finding that the decrease in multiple sclerosis attack rates in patients on IFN $\beta-1 b$ was not associated with any reduction in infection rate, ${ }^{12}$ that IFN $\beta-1 \mathrm{a}$ has no antiviral action. Further clarification must await the end of our double blind trial.

It has been claimed that the area or volume of Gd-DTPA enhancing lesions correlates with clinical worsening of multiple sclerosis. ${ }^{35-37}$ However, the methods of quantitation of enhancing multiple sclerosis lesions remains controversial. Various techniques have been employed in different studies, including manual tracing and area measurement, ${ }^{38} 39$ maximum diameter measurements of lesions, ${ }^{40}$ arbitrary scoring systems, ${ }^{18}$ and pixel mapping. ${ }^{41}$ In the last technique, enhancing voxels were counted at the subjective discretion of the investigator. ${ }^{40}$ Our technique employing 3D acquired MRI data was less biased and more reproducible in that the threshold for voxel enhancement was established statistically from normal appearing white matter. Sampling regions in the surrounding normal appearing white matter to establish a threshold intensity for each lesion allowed us to ignore the potential problem of fluctuations in the magnetic field intensity. Further, any small abnormalities present in the normal appearing white matter together with our threshold value of mean plus three SD provides a conservative underestimate of enhancing tissue volume.

In the present study there was overall a significant increase in the volume of Gd-DTPA enhancing lesions at the time of serologically proved viral exposures compared with not at risk periods, but as only three out of the eight patients showed this increase in volume in at risk periods it is difficult to know whether this was a chance finding or whether it may possibly be related to a treatment effect, as two thirds of the patients were randomised to active treatment with IFN $\beta-1$ a and one third to placebo. Other studies have shown that the introduction of IFN $\beta-1 b$ therapy is associated with a pronounced reduction in disease activity on Gd-DTPA enhanced MRI. ${ }^{42}$ If our data reflect more than a chance association, they may suggest a link between positive viral serology and MRI evidence of breakdown of the blood-brain barrier. Furthermore, the reduction of this breakdown may be associated with antiviral or immune modulating effects of IFN $\beta-1$ a. Alternatively, other patients may have had changes in enhancing lesion load on spinal MRI, or changes that were too small to detect with our MRI technique. Larger studies with more frequent scanning and greater MRI resolution may solve this problem. The lack of a similar effect on MRI at the times of diary reported symptomatic URTI could be due to overreporting of viral infections by our patients. The average annual rate of symptomatic URTI in our study (2.4 per year) was high and only $12.5 \%$ were serologically confirmed. By contrast, Narod et al reported an average annual rate of $1.6,{ }^{11}$ and Sibley et al rates of 0.79 to 1.22 in patients with high and low multiple sclerosis attack rates, respectively. ${ }^{9}$

The results reported here confirm a temporal association between symptomatic URTI and clinical exacerbations of multiple sclerosis and suggest that the relative risk of exacerbations is even higher when viral infection is serologically confirmed. In addition, we have found some evidence which may indicate that increased blood-brain barrier breakdown and inflammation occur particularly during periods of viral-induced immune stimulation. This might have implications for understanding the mechanism of action of IFN $\beta$ in RRMS and help in the development of future treatment aimed at preventing exacerbations of multiple sclerosis.

This study was carried out during trial protocols 6789 and 6954 with the support of Ares-Serono. Thanks are due to Vicki Orpe (MS trial coordinator) and to Steve Evans and his colleagues in the MRI Unit at University Hospital, Nottingham for help in data collection.

1 Sadovnick AD, Armstrong H, Rice GPA, et al. A population based study of multiple sclerosis in twins: update. Ann Neurol 1993;33:281-5.

2 French Research Group on Multiple Sclerosis. Multiple sclerosis in 54 twinships: concordance rate is independent of zygosity. Ann Neurol 1992;32:724-7.

3 Poser CM. The epidemiology of multiple sclerosis: a general overview. Ann Neurol 1994;36(suppl 2):S180-93.

4 Elian M, Nightingale S, Dean G. Multiple sclerosis among the United Kingdom-born children of immigrants in Greater London. I Neurol Neurosurg Psychiatry 1990;53: 906-11.

5 Kennedy PGE, Steiner I. On the possible viral aetiology of multiple sclerosis. $Q \mathcal{F}$ Med 1994;87:523-8.

6 Allen I, Brankin B. Pathogenesis of multiple sclerosis-the immune diathesis and the role of viruses. $\mathcal{F}$ Neuropathol Exp Neurol 1993;52:95-105.

7 Tienari PJ. Multiple sclerosis: multiple etiologies, multiple Tienari PJ. Multiple sclerosis: mutip
genes? Ann Med 1994;26:259-67.

8 Souberbielle BE, Sziwlowski PWS, Russell WC. Is there a case for virus aetiology in multiple sclerosis? Scot Med $\mathcal{F}$ $1995 ; 40 ; 55-62$. 
9 Sibley WA, Bamford CR, Clark K. Clinical viral infections and multiple sclerosis. Lancet $1985 ; \mathrm{i}: 1313-15$.

10 Andersen 0, Lynger PE, Bergstrom T, et al. Viral infections trigger multiple sclerosis relapses: a prospective seroepidemiological study. 7 Neurol 1993;240:417-22.

11 Narod S, Johnson-Lussenburg CM, Zheng Q, et al. Clinical viral infections and multiple sclerosis. Lancet 1985; ii $165-6$

12 Panitch HS. Influence of infection on exacerbations of multiple sclerosis. Ann Neurol 1994 36(suppl):S25-8.

13 Sibley WA, Foley JM. Infection and immunisation in multiple sclerosis. Ann NY Acad Sci 1985;122:457-68.

14 Barkhof F, Scheltens P, Frequin STFM, et al. Relapsing remitting multiple sclerosis: sequential enhanced MR imaging $v$ clinical findings in determining disease activity. AfR Am f Roentgenol 1992;159:1041-7.

15 McFarland HF, Frank JA, Albert PS, et al. Using gadolinium-enhanced magnetic resonance imaging to 1992;32:758-66.

16 Capra R, Marciano N, Vignolo LA, et al. Gadoliniumpenteic acid magnetic resonance imaging in patients with relapsing

17 Willoughby EW, Grochowski E, Li DKB, et al. Serial magnetic resonance scanning in multiple sclerosis: a second prospective study in relapsing patients. Ann Neurol 1989;25:43-9.

18 Thompson AJ, Miller D, Youl B, et al. Serial gadoliniumenhanced MRI in relapsing/remitting multiple sclerosis of varying disease duration. Neurology 1992;42:60-3.

19 Poser CM, Paty D, Scheinberg L, et al. New diagnostic criteria for multiple sclerosis: guidelines for research protocols. Ann Neurol 1983;13:227-31.

20 Anderson MJ, Higgins PG, Davis LR, et al. Experimental parvoviral infection in humans. F Infect Dis 1985;152: 257-65.

21 Kermode AG, Thompson AJ, Tofts P et al. Breakdown of the blood brain barrier precedes symptoms and other MRI signs of new lesions in multiple sclerosis. Pathogenic and signs of new lesions in multiple sclerosis. Patho
clinical implication. Brain 1990;113:1477-89.

22 Souberbille BE, Swingler RJ, Davidson DLW, et al. Western blotting analysis in patients with MS using human blood vessels as antigens. Acta Neurol Scand 1992;86;397-402.

23 Small JA, Scangos GA, Cork L, et al. The early region of human papovavirus JC induces dysmyelination in transgenic mice. Cell 1986;46;13-8.

24 Field EC, Cowshall S, Narang HK, et al. Viruses in multiple sclerosis? Lancet 1972;ii:280-1.

25 ter Meulen V, Koprowski H, lwasaki Y, et al. Fusion of cultured multiple sclerosis brain cells with indicator cells; presence of nucleocapsids and virions and isolation of parainfluenza type virus. Lancet 1972;ii: $1-5$.

26 Cook SD, Dowling PC, Russell WC. Multiple sclerosis and canine distemper. Lancet 1978;i:605-6.

27 Goswami KKA, Morris RJ, Rastogi SC, et al. A neutralizing monoclonal antibody against a paramyxovirus reacts with a brain antigen. F Neuroimmunol 1985;9:99-108.
28 Koprowski H, DeFreitas EC, Harper ME, et al. Multiple sclerosis and human $\mathrm{T}$ cell lymphotropic retroviruses. Nature 1985;318;154-60.

29 Wamer HB, Carp RI. Multiple sclerosis-an Epstein-Barr virus hypothesis. Med Hypotheses 1988;25:93-7.

30 Burks JS, Devald BL, Jankovsky LD, et al. Two coronaviruses isolated from the central nervous system tissue of two multiple sclerosis patients. Science 1980;209:933-4.

31 Wucherpfennig KW, Sette A, Southwood S, et al. Structural requirements for binding of an immunodominant myelin basic protein peptide to the DR2 isotypes and for its recognition by human T cell clones. F Exp Med 1994;179:27990.

32 Perron $\mathrm{H}$, Suh $\mathrm{M}$, Lalande $\mathrm{B}$, et al. Herpes simplex virus ICPO and ICP4 immediate early proteins strongly enhance expression of a retrovirus harboured by a leptomeningeal cell line from a patient with multiple sclerosis. $\mathcal{f}$ Gen Virol 1993;74:65-7.

33 Fox JP, Hall CE. Viruses in families. Littleton; PSG, 1980:82.

34 Miller AE, Morgante LA, Buchwald LY, et al. A multicentre, randomized, double-blind, placebo-controlled trial of influenza immunization in multiple sclerosis. Neurology 1997;48:312-4.

35 Filippi M, Horsfield MA, Campi A, et al. Resolutiondependent estimates of lesion volumes in magnetic resonance imaging studies of the brain in multiple sclerosis. Ann Neurol 1995;38:749-54.

36 van Walderveen MAA, Barkhof F, Hommes OR, et al. Correlating MRI and clinical disease activity in multiple sclerosis: relevance of hypointense lesions on short-TR/ short-TE (T1-weighted) spin-echo images. Neurology 1995;45:1684-90.

37 Frank JA, Stone LA, Smith ME, et al. Serial contrastenhanced magnetic resonance imaging in patients with early relapsing-remitting multiple sclerosis: implications for treatment trials. Ann Neurol 1994;36:S86-90.

38 Koopmans RA, Li DK, Grochowski E, Cutler PJ, et al. Benign versus chronic progressive multiple sclerosis: magnetic resonance imaging features. Ann Neurol 1989;25:74-

39 Kastrukoff LF, Oger JJ, Hashimoto SA, et al. Systemic lymphoblastoid interferon therapy in chronic progressive multiple sclerosis. I. Clinical and MRI evaluation. Neurology 1990;40:479-86.

40 Wiebe S, Lee DH, Karlik SJ, et al. Serial cranial and spinal cord magnetic resonance imaging in multiple sclerosis. Ann Neurol 1992;32:643-50.

41 Smith ME, Stone LA, Albert PS, et al. Clinical worsening in multiple sclerosis is associated with increased frequency and area of gadopentetate dimeglumine-enhancing magnetic resonance imaging lesions. Ann Neurol 1993;33:480-9.

42 Stone LA, Frank JA, Albert PS, et al. The effect of interferon- $\beta$ on blood-brain barrier disruptions demonstrated by contrast-enhanced magnetic resonance imaging in relapsing-remitting multiple sclerosis. Ann Neurol 1995; 37:611-9. 